\title{
A FUZZY-BASED CONGESTION CONTROLLER FOR CONTROL AND BALANCE CONGESTION IN GRIED-BASED WSN
}

\author{
Ali Dorri and Seyed Reza Kamel \\ Department of Computer Engineering, Mashhad Branch, \\ Islamic Azad University, Mashhad, Iran \\ Alidorri@mshdiau.ac.ir \\ Rezakamel@computer.org
}

\begin{abstract}
A Wireless Sensor Network (WSN) is deployed with a large number of sensors with limited power supply in a wide geographically area. These sensors collect information depending on application. The sensors transmit the data towards a base station called sink. Due to the relatively high node density and source-to-sink communication pattern, congestion is a critical issue in WSN. Congestion not only causes packet loss, but also leads to excessive energy consumption as well as delay. To address this problem, in this paper we propose a new fuzzy logic based mechanism to detect and control congestion in WSN. In the proposed approach, a Monitor Node for each grid in congestion candidate region performs a fuzzy control to avoid increasing congestion. Fuzzy controller's inputs are continually fetched from the network by the Monitor Node. Simulation results show that our approach has higher packet delivery ratio and lower packet loss than existing approaches.
\end{abstract}

\section{KEYWORDS}

Fuzzy Logic, Wireless Sensor Network, Congestion Control, Packet Lost.

\section{INTRODUCTION}

A Wireless Sensor Network (WSN) consists of spatially distributed autonomous wireless sensor nodes to cooperatively monitor physical or environmental conditions, such as temperature, sound and pressure. In addition, WSN is a network made of hundreds or thousands of sensor nodes which are densely deployed in hazardous/unattended environment with capability of sensing, computing and sending information wirelessly to the base station (also called sink) via neighbour nodes. Figure 1 shows a WSN that collect information and send it to the sink. 


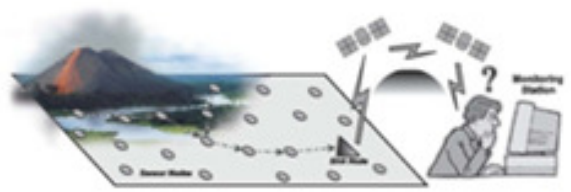

Figure 1. Wireless Sensor Network [1]

In WSN special applications, once an event occurs, a sudden surge of data traffic will be triggered by all sensor nodes in the event area, which may easily lead to congestion. Congestion in WSN has a negative impact on network performance. It increases the packet loss, end-to-end delay and wastes nodes energy. When a packet is lost, source node must retransmit it again. Therefore, node's energy is wasted and network lifetime will be decreased [2,3]. However, some characteristics of WSN, such as constrained resources and leak of centralized coordination, make the congestion problem in WSN more challengeable than any other networks. In WSN all nodes send their sensed data to the sink. This flow of packet (also called source-to-sink traffic), increase congestion probability and energy consumption in nodes near the sink. The reason is that, the neighbouring nodes of the sink should forward other nodes packets in addition to their own traffic $[4,5]$.

To address these challenges, we present a fuzzy congestion control. In the proposed approach, the network is divided into grids by the sink. Then, the sink specifies congestion candidate areas by use of a calculated threshold. In each congestion candidate area, a Monitor Node (MN) uses a fuzzy controller to detect and avoid congestion in its grid. Congestion level of each grid maybe different from other grids and if the congestion level of any congestion candidate area reaches to the acting level, the Border Node forwards packets out of the congested area. Therefore, congestion and packet loss will be decreased. Simulation results show that the presented fuzzybased system decreases the packet loss in congested area and increases packet delivery ratio to the sink. The remainder of this paper is organized as follows: section 2 presents a literature review of the congestion detection and control mechanisms. Section 3 provides detailed description of the proposed fuzzy controller approach. Performance evaluation of the proposed approach is presented in section 4 . Finally section 5 concludes and discusses the future directions of this research.

\section{RELATED WORKS}

In literature many congestion control schemes have been proposed for WSN. Congestion control schemes for WSN either focus on MAC layer or on both MAC and network layer [6]. Authors in [7] presented an approach based on a threshold. This threshold refers to ratio of received packets to serviced packets. In this approach each node has a priority. To detect congestion, both threshold and priority of nodes are influenced. Proposed approach is efficient in term of Quality of Service (QOS) as it sends data through multipath. Authors in [8] presented a Medium Access Control (MAC) technique to coordinate the access of nodes to the shared medium. It uses the queue buffer length of the sensor nodes to estimate the congestion. Then the traffic dynamically disseminates along with classifying nodes into different priority classes to provide a congestionfree routing path to the destination with improved QOS. In addition, it uses multiple forwarder traffic diffusion, which has advantages like increasing network reliability and reducing congestion. In [9] an optimal routing algorithm that allows optimizing transmission between the peripheral nodes and central node is presented, in order to increase the residual energy of the network. This protocol only aims to provide routing fidelity and does not address time transmission requirements. Authors in [10] presented a cross-layer congestion controller that has three parts: 1) multipath routing 2) adjusted ratio 3) application oriented design. In this algorithm, each node has multiple downstream nodes to be transmitted. The probability of forwarding nodes 
and the rate of sending packets can be dynamically adjusted according to the congestion state. Authors in [11] presented a novel approach based on bird's behaviour. The proposed approach is simple to implement at the individual node, involving minimal information exchange. In addition, it displays global self-properties and emergent behaviour, achieved collectively without explicitly programming these properties into individual packets. Performance evaluations show the effectiveness of the proposed Flock-based Congestion Control (Flock-CC) mechanism in dynamically balancing the offered load by effectively exploiting available network resources and moving packets to the sink. Furthermore, Flock-CC provides graceful performance degradation in terms of packet delivery ratio, packet loss, delay and energy consumption under low, high and extreme traffic loads. In addition, the proposed approach achieves robustness against failure and also has scalability in different network sizes and outperforms typical conventional approaches.

\section{THE PROPOSED APPROACH}

In previous section, we presented a literature review on congestion controller mechanisms in WSN. In this section, we briefly discuss our fuzzy-based congestion controller. Fuzzy system is used in order to increase accuracy of the congestion controller system. Fuzzy controllers convert crisp inputs to fuzzy based inputs, then by using a rule base, fuzzy system determines an action as the main output. The basic idea of a fuzzy controller is presented in Figure 2.

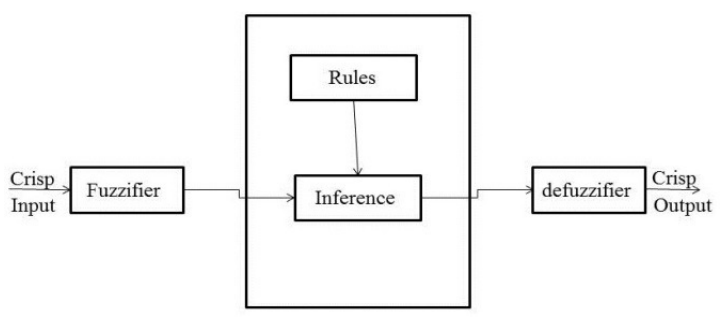

Figure 2. Fuzzy Controller System Functionality

In presented approach, fuzzy logic controller is considered as the kernel of the algorithm. It is associated with the Monitor Nodes (MNs) to detect congestion, based on information which comes from the network. In the propose approach, MN continuously monitors its grid and fetches fuzzy controller's metrics. Therefore, the fuzzy system calculates congestion level in each grid continuously and dynamically. We describe our fuzzy-based congestion controller in three phases that are as follows:

1) Congestion candidate generation

2) Congestion identification

3) Generating new phase

We discuss each phase briefly in the rest of this section.

\subsection{Congestion Candidate Generation}

After the sensors establishment, the sink uses nodes geographically information in order to divide network to some equal grids. In some applications of WSN, sensors deployed randomly in order to monitor environment and as the grids are equal in area, number of sensors in each grid maybe different from other grids. After dividing the network into grids, sink allocates an ID to each grid called Grid_ID. Then, it sends the Grid_ID of each grid to its members. The next step is choosing the Monitor Node (MN). Responsibility of the MN is to monitor its own grid continuously in order to detect congestion. Sink choose the sensor with highest reminded energy as the MN. In 
the case that two sensors have the same reminded energy, the nearest sensor to the sink is chosen as the MN. The MN needs to perform some monitoring works that consumes energy. Therefore, the sensor with highest reminded energy is chosen as the MN.

The presented fuzzy system uses each grid's density in order to detect congestion areas. Therefore, the first duty of each MN is to calculate its grids density. Whoever, at the beginning, sink knows the number of sensors in each grid, but in the case that a sensor dies as lack of energy, the MN must recalculate its grids density. When sensors energy reaches to the alarm level, it sends a packet for MN and aware MN of its death. The alarm levels energy is enough just for sending a packet to $\mathrm{MN}$.

In order to calculate grids density, the MN put Grid_ID in a packet and broadcast it for its neighbours. Each sensor that receives the packet compares its own Grid_ID with the received Grid_ID. If both are the same, the sensor sends a replay to the MN and then rebroadcasts packet. Sensor drops the packet, either if it received the same packet before or if packet's Grid_ID is not the same as node's Grid_ID.

Each MN sends the density of its grid to the sink. After receiving all grids densities, sink calculates the average of densities and uses it as a threshold for defining congested areas. Each grid with higher density than the calculated threshold is marked as the congested candidate area. If any change in number of sensors happened, the MN sends its new density for the sink. Sink recalculates the new threshold and updates congested candidate regions. High density regions have higher congestion probability and higher collision and packet lost rate. A summary of this phase is shown in Figure 3.

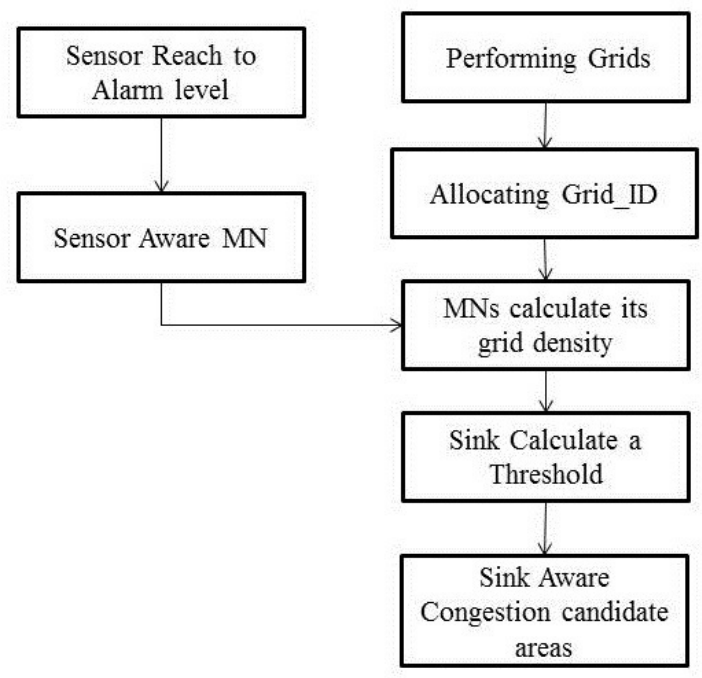

Figure 3. Steps for Congested Region discovery

\subsection{Congestion Identification}

In each congestion candidate grid, the $\mathrm{MN}$ has responsibility for detecting the congestion. In each MN there is a fuzzy-based system, which controls the congestion in grid and balance traffic in order to reduce the congestion. Fuzzy system uses three different metrics in order to decrease congestion and reduce its effects. Fuzzy controller for each $\mathrm{MN}$ is shown in Figure 4. MN continuously calculates three input parameters and then uses fuzzy logic to determine specific 
level of congestion. Based on the congestion level, either packets forward through the grid or through the relay nodes.

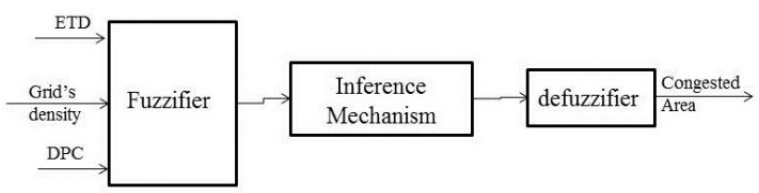

Figure 4. The Proposed Fuzzy Logic Controller

Three fuzzy input parameters are as follows:

ETD (Extended Transmission Delay): The ETD metric is the transition time that is required to transmit a packet to the next hop. The metric is calculated using the convex combination presented in Formula.1.

$\operatorname{ETD}(t)=n * \operatorname{nalnV}(t)+\left(1-\omega^{2} * \operatorname{FTn}(t-1) \quad\right.$ Formula.1

In this formula, Delay(t) determines delay in time $t$, and $\alpha$ is weight value. $\alpha$ is used to determine the priority of delay or previous ETD.

Grid's Density: High density of nodes in each grid can cause more congestion. In addition, grids density maybe increased or decreased during network lifetime. The reason is that a sensor may die or new sensors may add to the network. Number of sensors in each grid has a direct effect on congestion. Therefore, number of sensors is an important metric in the proposed fuzzy system.

DPC (Dropped Packets): Dropped packets, refers to number of lost packets in each grid. Packet loss is the result of congestion. Therefore, increasing packet loss means increasing congestion.

These parameters have membership functions that are presented in Figure 5. By use of these membership functions, a rule base is designed for fuzzy system. When MN calculates inputs, fuzzy system applies them in the rule base. Fuzzy system output determines three different actions that are as follows:

Action1) relay all packets through the grid

Action2) relay half of the packets through the grid and half through the relay nodes

Action3) relay all packets through the relay nodes

We will discuss the relay nodes and these actions in the next phase. Using presented mechanism in this phase, fuzzy controller detects congestion and try to reduce the packets in the congested area. 


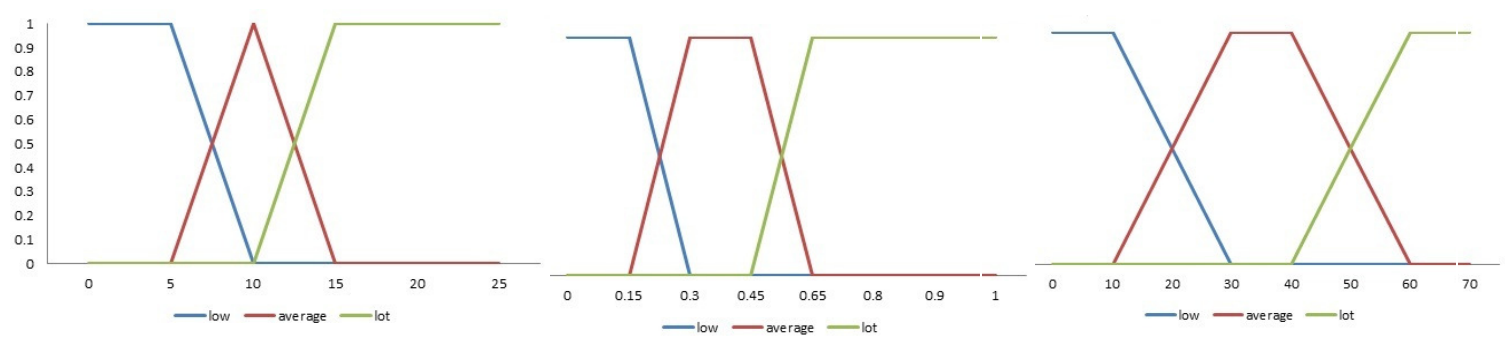
a) Grids Density
b) ETD
c) Dropped packets

Figure 5. Membership Functions Diagrams

\subsection{Generating New Path}

When fuzzy system determines the action, the MN performs the specified action. If there is no congestion, output will be action1. In this case, there is no need for the MN to perform any action. But for other two actions, the MN must informs its grid's Border Node (BN) about the congestion level. At the first time, the MN sends a packet to the sink and asks for its BN's ID. The sink selects the last node in grid as the BN. The detail of selecting BN is presented in [13]. Figure 6 shows the position of BNs and MNs in the network. After selecting the BN, the sink informs the $\mathrm{MN}$ of its grids BN. BN has the responsibility of relaying the packets either through the grid or through the relay nodes. The BN relay packets based on action level and this will continue until congestion level in the grid reduce to action1. When the BN gets informed of any changes in congestion level by the MN, it sends packets through relay path based of congestion level. Since radio frequency of relay nodes is different from ordinal sensors, sending packet using relay path has no effect on congestion in the grid. Sink selects the best relay path for BN based on [13].

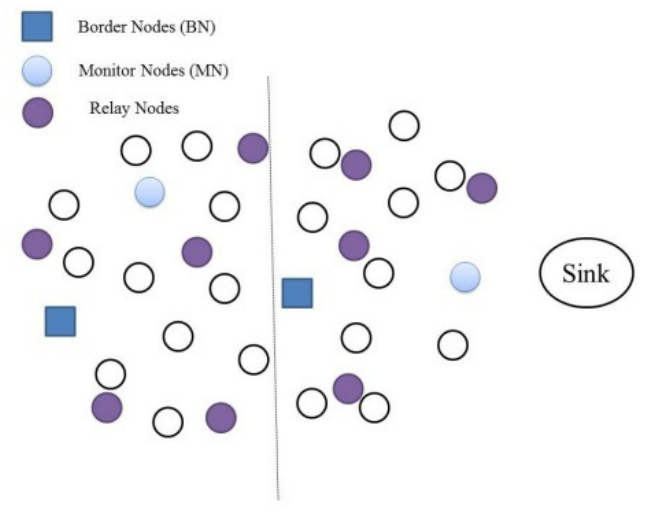

Figure 6. Example of $\mathrm{BN}$ and $\mathrm{MN}$ Assignments

In this section, we briefly discussed our proposed approach. A summary of the proposed approach is shown in Figure 7. 


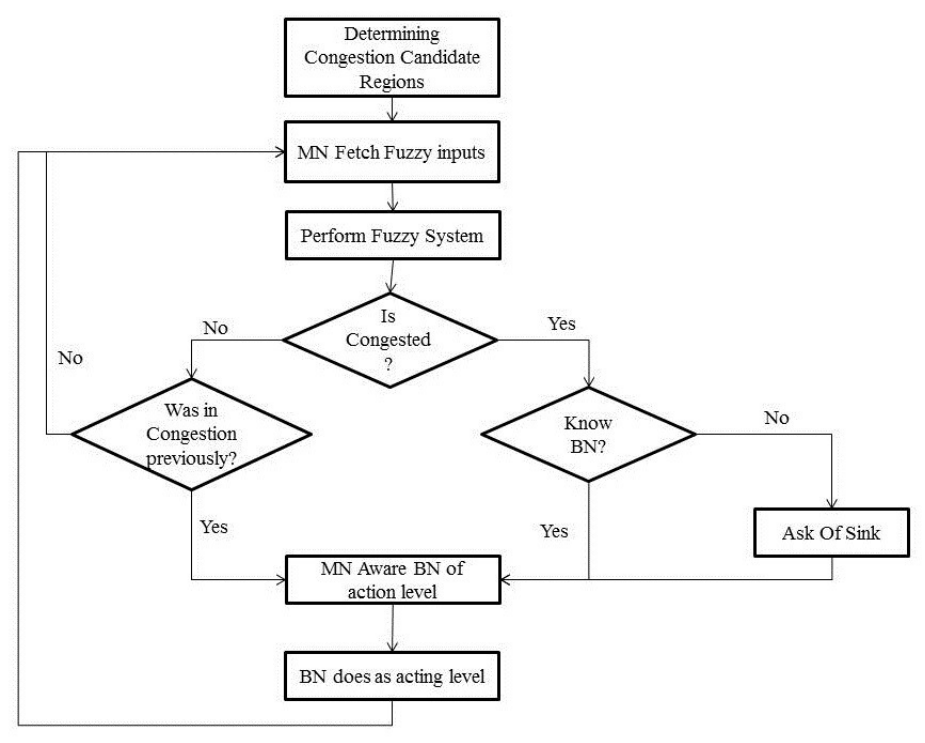

Figure 7. The Proposed Approach

\section{SiMULATION RESULTS}

To show the advantages of the proposed approach in compare with the Base Work (BW) [12], both approaches implemented using Opnet Modular 14.5 simulator. The simulation was performed using a WSN of size $300 \mathrm{~m} * 300 \mathrm{~m}$. Table 1, lists simulation parameters used in our study.

Both the BW and our approach were implemented using two different scenarios. For both scenarios two parameters have been measured and evaluated. These parameters are as follows:

Packet delivery: number of packets reached to the sink

Number of dropped packets: number of packet lost because of congestion in congestion candidate grids.

Table 1. Simulation Parameters.

\begin{tabular}{ll}
\hline Parameter & Value \\
\hline Simulation duration & $180 \mathrm{sec}$ \\
Number of nodes & 26 \\
Transmission range & $20 \mathrm{~m}$ \\
Traffic type & $\mathrm{CBR}(\mathrm{UDP})$ \\
Packet rate & 2 packets/sec \\
Data payload & $512 \mathrm{byte} / \mathrm{packet}$ \\
Number of relay nodes & 6 \\
\hline
\end{tabular}

The aim of the proposed fuzzy congestion controller is to detect and avoid increasing congestion in congestion candidate regions. When a packet is lost, it should be retransmitted by the source node which wastes node's energy and the network bandwidth.

Figure 8 presents packet delivery rate for both approaches. 


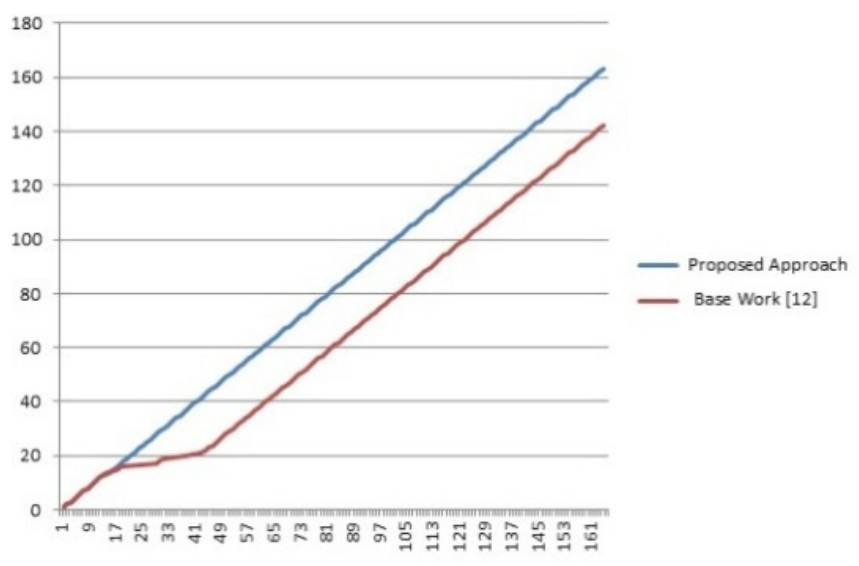

Figure 8. Packet Delivery Rate

In this figure horizontal axis refers to time and the vertical axis, to number of packets delivered to the sink. This figure shows the packet delivery ratio in the sink. Firstly in this figure, both curves have overlap as our fuzzy mechanism hasn't detected congestion yet. As fuzzy controller uses three metrics to detect congestion, after a while, it detects a level of congestion and starts to lead packets toward relay nodes, based on congestion level. As number of packet lost increased, BW starts to send all packets through relay nodes. Therefore, after a while both approach send packets through relay nodes and packet delivery ratio will be the same in the sink for both approaches.

Figure 9 shows the packet lost figure for both approaches.

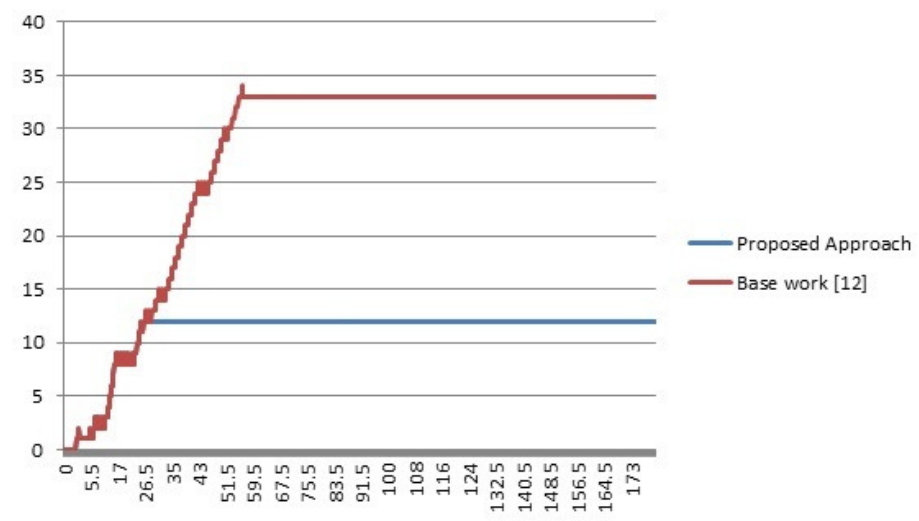

Figure 9. Packet Loss in Congested Grid rate

Vertical axis refers to packet lost in congested grid, and horizontal axis refers to time. Like Figure 8 , in this figure, firstly packet lost in both approaches is the same. After increasing packet lost, fuzzy system detects congestion and according to congestion level, it sends packets through either congested grid or relay nodes. As a result, congestion and packet lost in congested candidate grid will be decreased. Whoever, after a while, as both approaches relay packets through the relay nodes, packet lost is equal in both approaches.

Referred to simulation results, proposed approach increases packet delivery ratio in the sink and decreases packet lost in congested grids. In addition, fuzzy system controls the congestion and avoids growth in congestion. Also, fuzzy system is more flexible as it continuously fetches 
metrics from the network. By increasing the number of curves in membership functions of input metrics, the accuracy of system increases. As a result, number of actions and control ratio increases. When fuzzy system reaches to acting level, proposed approach controls congestion and helps the grid to balance traffic by leading some packets through relay paths.

\section{CONCLUSION AND FUTURE WORK}

Wireless Sensor Network (WSN) is a set of sensor nodes, which are distributed in an area. In WSN all sensor nodes sends their packets hop-by-hop to the sink, therefore, nodes nearer to the sink should forward their own packets and other nodes packets. This feature of WSN, increases congestion and packet lost in nodes, especially in nearer nodes to the sink. Using congestion controller mechanisms can avoid or control congestion in WSN. As a result of decreasing congestion, packet loss and energy consumption of the nodes will decrease. In this paper, we proposed a novel approach based on fuzzy logic to control and balance congestion in grid-based WSN. Presented approach uses three parameters that dynamically and continuously fetches from the network in order to detect congestion regions. When fuzzy system detects a level of congestion it relay some packets out of the grid in order to control and balance congestion. As our future work, we decided to make our work more energy aware as energy is the most important parameter in WSN.

\section{REFERENCES}

[1] L.A. Villas, A. Boukerche, H.S. Ramos, H.A.B.F. de Oliveira, "DRINA: A Lightweight and Reliable Routing Approach for In-Network Aggregation in Wireless Sensor Networks", IEEE Transactions on Computers, (Volume:62, Issue: 4 ), 2013.

[2] R. Annie Uthra, S.V. Kasmir Raja, " Energy Efficient Congestion Control in Wireless Sensor Network", Recent Advances in Intelligent Informatics Advances in Intelligent Systems and Computing Volume 235, pp 331-341, 2014.

[3] Sh. Borasia, V. Raisinghani," A Review of Congestion Control Mechanisms for Wireless Sensor Networks", Technology Systems and Management Communications in Computer and Information Science Volume 145, pp 201-206, 2011.

[4] C. Karakus, A.C. Gurbuz, B. Tavli," Analysis of Energy Efficiency of Compressive Sensing in Wireless Sensor Networks", Sensors Journal, IEEE (Volume:13, Issue: 5 ), 2013.

[5] C. Sergiou, V. Vassiliou, "Study of lifetime extension in wireless sensor networks through congestion control algorithms", IEEE Symposium on Computers and Communications (ISCC), 2011.

[6] J. Zhao, L. Wang, S. Li, X. Liu, " A Survey of Congestion Control Mechanisms in Wireless Sensor Networks", in Sixth International Conference on Intelligent Information Hiding and Multimedia Signal Processing (IIH-MSP), 2010.

[7] C. Wang, B. Li, K. Sohraby, M. Daneshmand, "Upstream congestion control in wireless sensor networks through cross-layer optimization". IEEE Journal on Selected Areas in Communications, (Volume:25, Issue: 4 ).

[8] U.S. Visweswaraiya, K.S. Gurumurthy, " A Novel, Dynamic Data Dissemination [D3] Technique for Congestion Avoidance/Control in High Speed Wireless Multimedia Sensor Networks", Fifth International Conference on Computational Intelligence, Modelling and Simulation (CIMSim), 2013.

[9] E. Hajian, K. Jamshidi, A. Bohlooli, "Improve energy efficiency routing in WSN by using automata", International Journal of Ad hoc, Sensor \& Ubiquitous Computing (IJASUC) Vol. 1, No.2, 2010.

[10] Li. Zilong, W. Zou, T. Qi, ” A cross-layer congestion control strategy in wireless sensor network”, in 4th IEEE International Conference on Broadband Network and Multimedia Technology (IC-BNMT), 2011.

[11] P. Antoniou, A. Pitsillides, T. Blackwell, A. Engelbrecht, L. Michael," Congestion control in wireless sensor networks based on bird flocking behavior", Computer Networks Volume 57, Issue 5, 7 April 2013, Pages 1167-1191. 
[12] H. Cha, K. Kim, S.Yoo, ” A node placement algorithm for avoiding congestion regions in wireless sensor networks", in Third International Conference on Ubiquitous and Future Networks (ICUFN), 2011.

[13] J. Mena, V. Kalogeraki, " Dynamic Relay Node Placement in Wireless Sensor Networks" in International Symposium on Applications and the Internet, SAINT ,2008.

\section{AUTHORS}

Ali Dorri received his B.S. degree in computer engineering from Bojnord University, Iran, in 2012, and now is student in M.S in software engineering in Mashhad branch, Islamic Azad University, Mashhad, Iran. His research interests cover Wireless Sensor Networks (WSN), Mobile Ad hoc Network (MANET) and specially Security challenges.

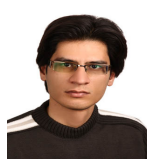

Dr. Seyed Reza Kamel Tabbakh is with the Department of Software Engineering, Faculty of Engineering, Islamic Azad University - Mashhad branch, Mashhad, Iran. He received his $\mathrm{PhD}$ in communication and network engineering from University Putra Malaysia (UPM) in 2011. He received his BSc and MSc in software engineering from Islamic Azad University, Mashhad branch and Islamic Azad University, South Tehran branch, Iran in 1999 and 2001 respectively. His research interests include IPv6 networks,

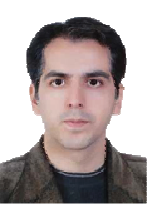
routing and security. During his studies, he has published several papers in International journals and conferences. 